\title{
Critical nucleus charge in a superstrong magnetic field
}

\author{
S.I. Godunov ${ }^{1,2, a}$, S.I. Glazyrin ${ }^{1,2,3, b}$, B. Machet ${ }^{4,5, c}$, and M.I. Vysotsky ${ }^{1,6, d}$ \\ ${ }^{1}$ Institute for Theoretical and Experimental Physics, Moscow, 117218, Russia \\ ${ }^{2}$ Novosibirsk State University, Novosibirsk, 630090, Russia \\ ${ }^{3}$ Dukhov Research Institute of Automatics (VNIIA), Moscow, 127055, Russia \\ ${ }^{4}$ Sorbonne Universités, LPTHE, Université P. et M. Curie, BP 126, 4, place Jussieu F-75252 Paris Cedex \\ 05, France \\ ${ }^{5}$ CNRS, UMR 7589, LPTHE, F-75005, Paris, France \\ ${ }^{6}$ National Research University Higher School of Economics, Myasnitskaya str., 20, Moscow 101978, Russia
}

\begin{abstract}
Due to strong enhancement of loop effects in a superstrong magnetic field $\left(B \gg m^{2} / e^{3}\right)$ the Coulomb potential becomes screened. This phenomenon dramatically changes the dependence of the electron energy levels on magnetic field. In particular, the freezing of energy levels occurs so the ground energy level of light ions can never reach the lower continuum (become critical), no matter how strong the field is.

Therefore, the magnetic field affects the critical nucleus charge $Z_{\mathrm{cr}}$ in two ways: i. it makes the electron movement essentially one-dimensional diminishing the value of $Z_{\mathrm{cr}}$; ii. it makes the potential weaker increasing the value of critical charge.

The phenomenon of critical charge itself is also discussed.
\end{abstract}

\section{Introduction}

The Dirac equation cannot be solved for the electron in the potential of the pointlike Coulomb center with charge $Z>137$. However, it can be done if the finite size of a nucleus is taken into account (see, for example, [1-3]; more references can be found in [4]). At $Z=Z_{\mathrm{cr}} \sim 170$ ground energy level reaches lower continuum ( $\varepsilon=-m$, where $m$ is the electron mass) and spontaneous electron-positron pair production occurs. Electrons occupy ground level and one can observe two free positrons.

Since the value of the critical charge is too large, it seems impossible to produce such a nucleus for a sufficiently long period of time. There were searches for this effect in heavy ion collisions, but no evidence was found so far.

Later it was discovered that the value of critical nucleus charge can be diminished by external magnetic field. Analytical results for the limit of strong magnetic fields were found in [5]. More accurate results taking into account higher Landau Levels were found numerically in [6]. In those papers the potential was taken to be the Coulomb one.

\footnotetext{
ae-mail: sgodunov@itep.ru

be-mail: glazyrin@itep.ru

ce-mail: machet@lpthe.jussieu.fr

$\mathrm{d}_{\mathrm{e}-\mathrm{mail}}$ : vysotsky@itep.ru
} 
Radiative corrections to the Coulomb potential are usually small and lead to small shifts in atomic spectra. However, in the presence of external fields these corrections can be significantly enhanced. For example, in an external magnetic field vacuum polarization in one loop is strongly enhanced and proportional to magnetic field strength $B$. It was discovered by Shabad and Usov [7, 8] (see also [9]) that this enhancement leads to the significant modification (screening) of the Coulomb potential for $B \gtrsim m^{2} / e^{3}$ where $m$ and $e$ are electron mass and charge ${ }^{1}$. The potential was found numerically along the magnetic field on the line going through the charge and in the direction transverse to the magnetic field in the plane containing the charge. Analytical asymptotics were also found in there.

Interpolation (but still quite accurate) formula for the polarization operator in a superstrong magnetic field was suggested by Vysotsky [10]. With the help of this formula the potential for $^{2} z=0$ with arbitrary $\rho$ and for $\rho=0$ with arbitrary $z$ was calculated analytically [11, 12]. It was found [7, 8, 12] that for $z \gg 1 / m$ the equipotential lines are ellipses, though the potential in the mid-range distances, $r=\sqrt{\rho^{2}+z^{2}} \lesssim 1 / m$, was still unknown. In Fig. 1 in [12] the equipotential lines were approximated by ellipses everywhere. The numerical results for the potential in all directions were obtained in [13].

In Section 2 the modification of the potential due to loop corrections is studied. In Section 3 the critical nucleus charge with the account of potential modification is considered. In Section 4 aspects of the critical nucleus charge phenomenon is discussed.

This talk is based on papers $[4,10-16]$.

\section{Modification of the Coulomb potential by external superstrong magnetic field}

In this section we briefly describe current analytical and numerical results on the modified potential. In the presence of an external superstrong magnetic field $\left(B \gg B_{0} \equiv \mathrm{m}^{2} / e \approx 4.4 \cdot 10^{13} \mathrm{G}\right)$ the contribution of vacuum polarization at one loop level to the polarization operator becomes greatly enhanced [17-20]):

$$
\Pi^{(2)}\left(k_{\perp}, k_{\|}\right)=-\frac{2 e^{3} B}{\pi} \exp \left(-\frac{k_{\perp}^{2}}{2 e B}\right) T(t),
$$

where $k=(0, \vec{k})=\left(0, \vec{k}_{\perp}, k_{\|}\right)$is the momentum of the external photon, $t \equiv k_{\|}^{2} / 4 m^{2}$ and

$$
T(t)=1-\frac{1}{\sqrt{t(1+t)}} \log (\sqrt{1+t}+\sqrt{t}) .
$$

Here we took into account only Lowest Landau Level (LLL) contribution into polarization operator which should dominate. Contributions from higher loops are also omitted in what follows. With these approximations we follow $[7,8,10-12,15,18]$ where the arguments in favour of this approximation can be found.

The polarization operator enters the photon propagator leading to the modification of the pointlike charge potential (we consider elementary charge $e$ ):

$$
\Phi(\rho, z)=4 \pi e \int \frac{d^{2} k_{\perp} d k_{\|}}{(2 \pi)^{3}} \frac{e^{-i \overrightarrow{k_{\perp}} \vec{\rho}} e^{-i k_{\|} z}}{k_{\|}^{2}+k_{\perp}^{2}-\Pi^{(2)}\left(k_{\perp}, k_{\|}\right)} .
$$

\footnotetext{
${ }^{1}$ The Gauss system of units is used, $e^{2}=\alpha=1 / 137.035 \ldots$, therefore $m^{2} / e^{3}=B_{0} / \alpha$ where $B_{0} \equiv m^{2} / e \approx 4.4 \cdot 10^{13} \mathrm{G}$.

${ }^{2}$ We use cylindrical geometry $(\rho, \phi, z)$ with the pointlike charge located at $(\rho, z)=(0,0)$ and magnetic field directed along $z$ axis.
} 
After integration over the angle in the plane transverse to the magnetic field the following result was obtained $[7,8]$ (up to units and notations):

$$
\Phi(\rho, z)=\frac{e}{\pi} \int_{-\infty}^{\infty} d k_{\|} e^{-i k_{\|} z} \int_{0}^{\infty} d k_{\perp} \frac{k_{\perp} J_{0}\left(k_{\perp} \rho\right)}{k_{\perp}^{2}+k_{\|}^{2}+\frac{2 e^{3} B}{\pi} e^{-k_{\perp}^{2} / 2 e B} T\left(k_{\|}^{2} / 4 m\right)} .
$$

In [10] the interpolation formula for $T(t)$ was introduced:

$$
T(t) \approx \frac{2 t}{2 t+3} .
$$

It is rather simple and allows to perform analytical calculations, but at the same time it provides a very good accuracy (see [11] for details).

Using formula (5) the analytical expressions for $\Phi(0, z)$ and $\Phi(\rho, 0)$ were found $[11,12]$ (see also asymptotics in $[7,8])$ :

$$
\Phi(0, z)=\frac{e}{|z|}\left(1-e^{-|z| \sqrt{6 m^{2}}}+e^{-|z| \sqrt{(2 / \pi) e^{3} B+6 m^{2}}}\right)
$$

and for $B \gg 3 \pi m^{2} / e^{3}$ :

$$
\Phi(\rho, 0)= \begin{cases}\frac{e}{\rho} \cdot \exp \left(-\rho \sqrt{(2 / \pi) e^{3} B}\right), & \rho<l_{0}, \\ \frac{e}{\rho} \cdot \sqrt{\frac{3 \pi m^{2}}{e^{3} B}}, & \rho>l_{0},\end{cases}
$$

where $l_{0} \equiv \sqrt{\frac{\pi}{2 e^{3} B}} \ln \sqrt{\frac{e^{3} B}{3 \pi m^{2}}}$.

The potential at large distances, $z \gg 1 / m$, was found in $[7,8,12]:^{3}$

$$
\left.\Phi(\rho, z)\right|_{z \gg 1 / m}=\frac{e}{\sqrt{z^{2}+\rho^{2}\left(1+\frac{e^{3} B}{3 \pi m^{2}}\right)}},
$$

which means that equipotential lines at large distances are ellipses.

The numerical calculation of the initial integral (4) in all space was performed in [13]. The numerical results are in a good agreement with results for $\Phi(0, z)$ and $\Phi(\rho, 0)$ obtained in [7, 8]. Numerically obtained equipotential lines for $B=10^{4} B_{0}$ and $B=10^{5} B_{0}$ are shown in Fig. 1 . The central part of the pattern shown in Fig. 1b is magnified in Fig. 1c. We see that outer equipotential line has elliptic shape and it is in a good agreement with analythical results. The inner line is a circle with a good accuracy, as it should be (Yukawa law in all directions). One could expect that at mid-range distances lines should have ellipse-like shape too, but as we can see from the numerical results they are "eye-shaped" rather than elliptical. In Section 3 only analytical results (6), (7), and (8) are taken into account.

\section{Critical nucleus charge in the modified potential}

It was discovered by Oraevskiy, Rez, Semikoz [5] that the value of critical charge can be diminished by a magnetic field. They solved Dirac equation in the limit of a strong magnetic fields and obtained

\footnotetext{
${ }^{3}$ Within the effective Lagrangian approach (Euler-Heisenberg), corrections to the formula (8) were found in [21, 22].
} 


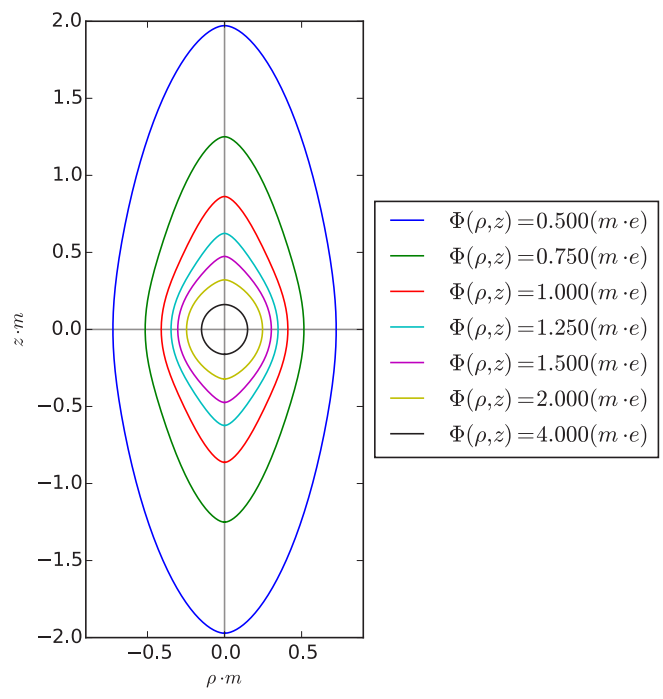

(a) $B=10^{4} B_{0}$

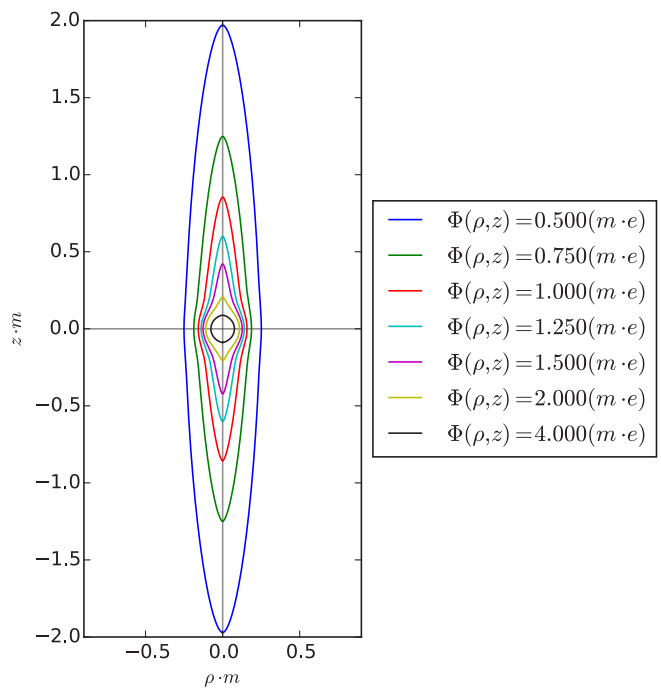

(b) $B=10^{5} B_{0}$ : Overview

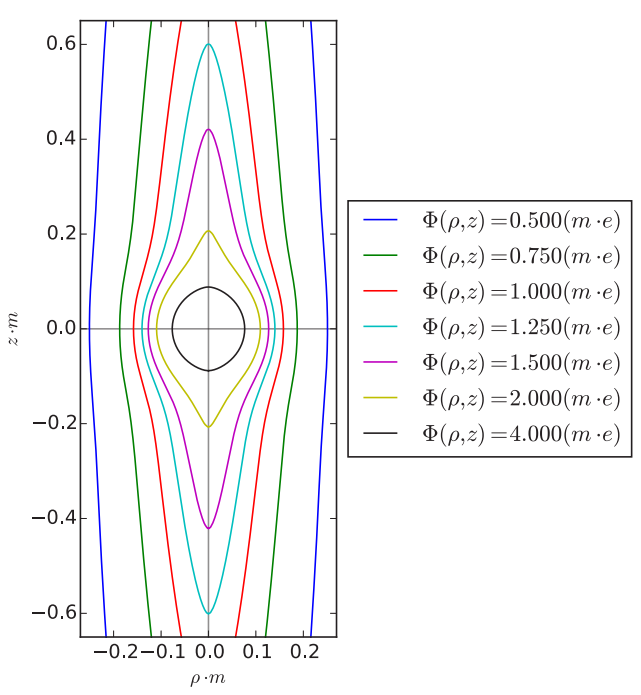

(c) $B=10^{5} B_{0}$ : Central part

Figure 1: The equipotential lines for $B=10^{4} B_{0}$ and $B=10^{5} B_{0}$. The values in the legend correspond to the equipotential lines from outer to the inner one.

the equation on the energy $\varepsilon$ of the electron moving in the potential of the nucleus with charge $Z$ :

$$
\begin{aligned}
Z e^{2} \ln \left(2 \frac{\sqrt{m^{2}-\varepsilon^{2}}}{\sqrt{e B}}\right)+\arctan \left(\sqrt{\frac{m+\varepsilon}{m-\varepsilon}}\right) & +\arg \Gamma\left(-\frac{Z e^{2} \varepsilon}{\sqrt{m^{2}-\varepsilon^{2}}}+i Z e^{2}\right)- \\
& -\arg \Gamma\left(1+2 i Z e^{2}\right)-\frac{Z e^{2}}{2}(\ln 2+\gamma)=\frac{\pi}{2}+n \pi,
\end{aligned}
$$


Table 1: Values of $\varepsilon_{0} / m$ for $Z=40$.

\begin{tabular}{|c||c|c|c|}
\hline$B / B_{0}$ & Eq. 9 & Numerical results & $\begin{array}{c}\text { Numerical results } \\
\text { with screening }\end{array}$ \\
\hline $10^{0}$ & 0.819 & 0.850 & 0.850 \\
$10^{1}$ & 0.653 & 0.667 & 0.667 \\
$10^{2}$ & 0.336 & 0.339 & 0.346 \\
$10^{3}$ & -0.158 & -0.159 & -0.0765 \\
$10^{4}$ & -0.758 & -0.759 & -0.376 \\
$2 \cdot 10^{4}$ & -0.926 & -0.927 & -0.423 \\
$\ldots$ & at $B / B_{0} \approx 2.85 \cdot 10^{4}, \varepsilon_{0}=-m$ & $\ldots$ \\
$10^{5}$ & - & - & -0.488 \\
$10^{6}$ & - & - & -0.524 \\
$10^{7}$ & - & - & -0.535 \\
$10^{8}$ & - & - & -0.538 \\
\hline
\end{tabular}

where $\gamma=0.5772 \ldots$ is the Euler constant. For the ground level at $\varepsilon>0$ one should take $n=0$, while for $\varepsilon<0$ it should be changed to $n=-1$.

According to (9) when the magnetic field increases the ground state energy goes down and reaches the lower continuum. The value of the magnetic field at which this happens is determined by the formula:

$$
\frac{B}{B_{0}}=2\left(Z_{\mathrm{cr}} e^{2}\right)^{2} \exp \left(-\gamma+\frac{\pi-2 \arg \Gamma\left(1+2 i Z_{\mathrm{cr}} e^{2}\right)}{Z_{\mathrm{cr}} e^{2}}\right) .
$$

For uranium $(Z=92)$ we get about $100 B_{0}$, for $Z=61$ it gives $10^{3} B_{0}$, for $Z=41$ it gives $2 \cdot 10^{4} B_{0}$.

The Dirac equation for electron both in an external magnetic field and in the Coulomb field of a nucleus was numerically solved in [6]. The exact solutions of [6] were compared with the approximate analytical ones of [5] (see (9)) in Figures 1 and 2 in [6].

To take screening into account we solved Dirac equation numerically. The details of this calculation can be found in [12]. The results for ground energy level for the ion with $Z=40$ are presented in Table 1. In the second and the third columns we compare analytical formula Eq. 9 and our numerical results without screening. They are in a very good agreement and we can see when ground energy level reaches lower continuum. But the situation is completely different when we turn the screening on: the ground energy level freezes at about minus a half of the electron mass. So the ion with charge 40 never becomes critical. In Figure 3 the results for different $Z$ are presented by green (dashed) line. We see that taking screening into account increases the value of magnetic field at which ions become critical. This increment is bigger for smaller $Z$ and ions with charge less than 50 do not reach criticality.

The ions with $Z \lesssim 55$ reach criticality in such a strong magnetic field that Landau radius $a_{H}$ becomes smaller than the size of the nucleus. That is why the finiteness of the nucleus radius should be taken into account.

Using analytical results (6), (7), and (8) the approximate expression for the potential of the nucleus with finite size has been found along the magnetic field (see Ref. [14] for the details). Then the Dirac equation was solved numerically in this potential. For calculations we took the nucleus radius to be 


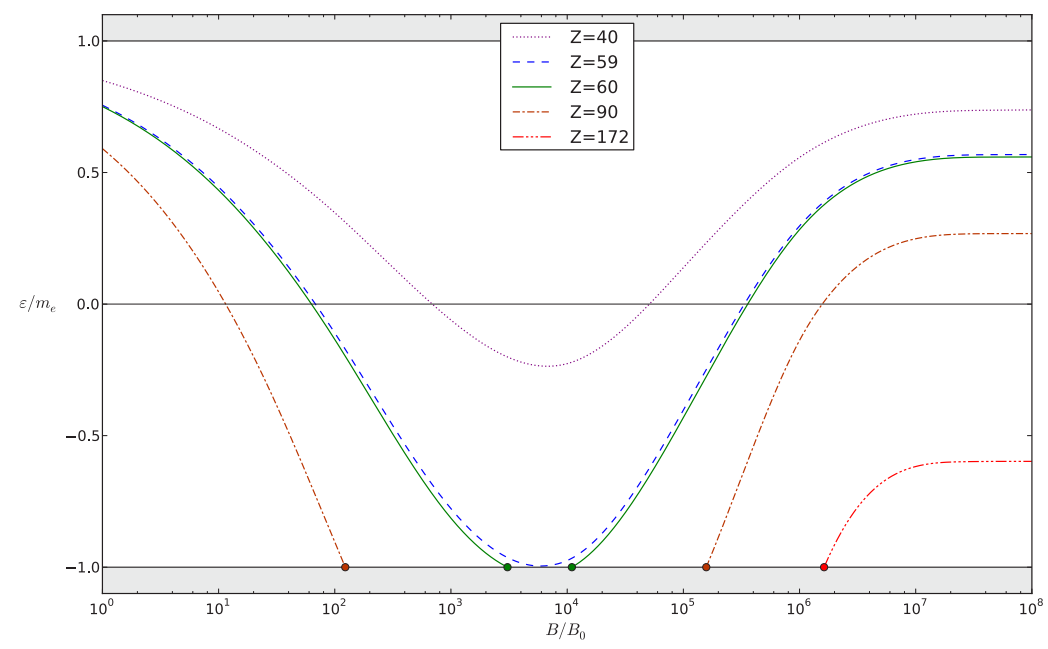

Figure 2: The dependence of the ground energy level on magnetic field for $Z=40,59,60,90,172$. The correspondence between charge $Z$ and color (linestyle) is shown in the legend.

$R_{Z}=r_{0} A^{1 / 3}$ with $r_{0}=1.1 \mathrm{fm}$ and $A=2.5 Z$. In Fig. 2 the dependence of the ground state energy on the magnetic field for $Z=40,59,60,90,172$ is shown. We see that ions with $Z<60$ never become critical while a nucleus with $Z=60$ is critical only within the small range of magnetic fields around $B \approx 10^{4} B_{0}$. For larger $Z$ the range of magnetic fields in which ions are critical becomes wider. Ions become critical at $B$ a little bit larger than the critical field for a pointlike nucleus. The rising of the ground energy level makes the ions noncritical for strong enough magnetic fields. Even an ion with $Z=172$ becomes noncritical for $B>1.6 \cdot 10^{6} B_{0}$ while it is critical for $B=0$. To estimate the value of the nucleus charge at which the "final", or "second", freezing energy reaches the lower continuum we demanded that the nucleus should be critical for $B=10^{8} B_{0}$ and found that it satisfied for $Z \geq 210$. It means that only ions with $Z \geq 210$ remains critical regardless of the value of the magnetic fields. In Fig. 3 the dependence of critical nucleus charge on $B$ is shown.

\section{Positron scattering on a supercritical nucleus}

In this section we want to discuss some aspects of the phenomenon of critical charge. In relatively recent paper [23] the scattering of positrons on a supercritical nuclei was studied and it was claimed that there is no spontaneous $e^{+} e^{-}$pair production from a supercritical nucleus. This made us to reexamine the physics of supercritical nuclei in paper [4] and in this section we briefly discuss the results obtained there.

The scattering of positrons on a supercritical nucleus has the spectacular resonance behavior discovered in $[2,3,23]$. In the paper [4] results with an exact dependence on the parameter $m \times R$ have been obtained on the both sheets of the complex energy plane in the form convenient for numerical evaluation. However one can hardly hope to study this phenomenon experimentally: even if a supercritical nucleus can be produced in heavy ions collisions, its life time will be so short that it makes impossible to scatter a positron on it, not to mention the still bigger challenge of making a target with supercritical nuclei. Let us note that since the elastic scattering matrix was found to be unitary 


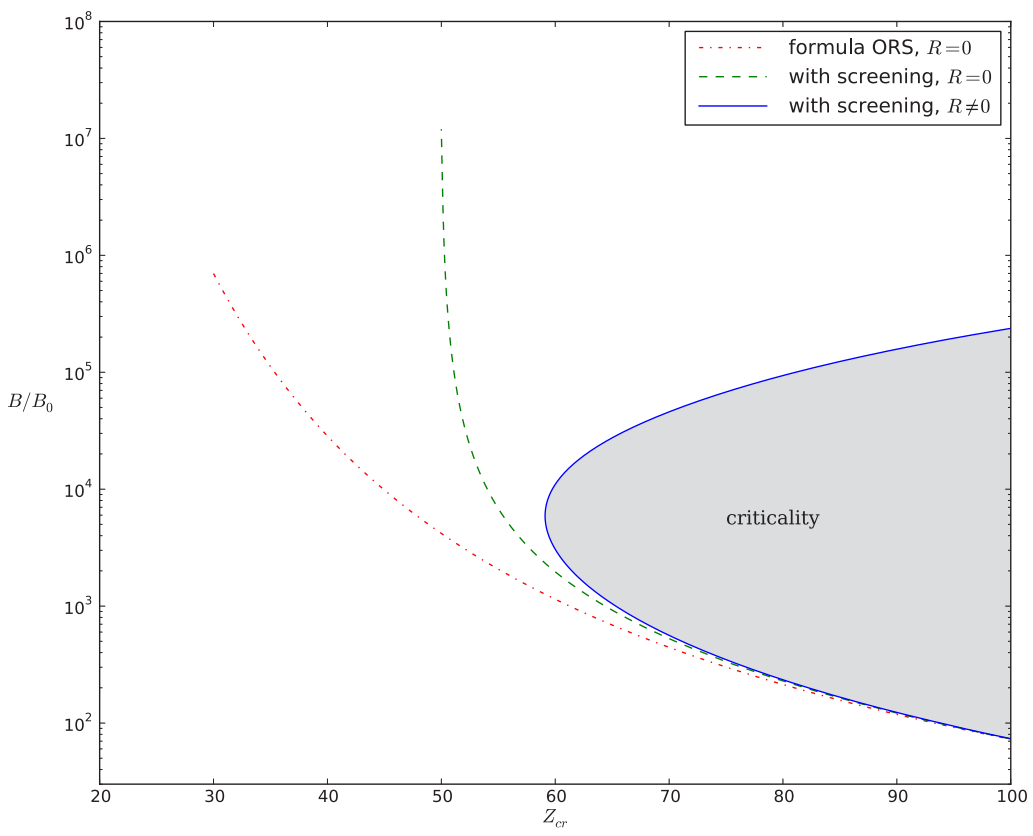

Figure 3: The values of magnetic fields at which the nuclei with charge $Z_{\mathrm{cr}}$ becomes critical: a) without screening according to eq. (9), dot-dashed (red) line; b) numerical results with screening for pointlike nucleus, dashed (green) line; c) numerical results which take finite size of the nucleus into account, solid (blue) line.

(the scattering phase is real) there are no inelastic processes in the positron scattering on supercritical nucleus.

More realistic is the hope to detect the emission of positrons from a short-lived supercritical nucleus eventually produced in heavy ions collisions. Indeed we do not agree with the claim made in the abstract of [23] (and in contradiction with [1] in particular) that the spontaneous production of $e^{+} e^{-}$ pairs from a supercritical nucleus does not occur. On the contrary, we believe that the resonance found in [23] in the system positron-supercritical nucleus is precisely the signal for pair production. It occurs when, as $Z$ grows, an empty electron level dives into the lower continuum of the Dirac equation. In the absence of the nucleus, this empty state in the lower continuum would just mean the presence of a positron. The presence of the nucleus makes the energy of this state complex, and its lifetime is precisely $1 / \gamma$, where $\gamma$ is the imaginary part of the energy. In this lapse of time, an electron from the sea with the same energy located far from the nucleus can penetrate in its vicinity. It partially screens the charge of the nucleus and, at the same time, an empty electron state arises in the Dirac sea. This is the positron which gets repulsed to infinity by the nucleus.

Let us suppose that solutions of the Dirac equation we get are approximately valid also when an electron screens nuclear potential, being embedded in the lower continuum. It means our solutions for the resonance energy and width are almost valid. It well can be so, since electric charge of one electron is small and it is situated far from nucleus, $r \approx 1 / \mathrm{m}$. So, the obtained width (imaginary part of energy) is the lifetime of positron in the vicinity of nucleus, which is already surrounded by diving electron. Therefore this is the lifetime of the system of nucleus, electron and positron with 


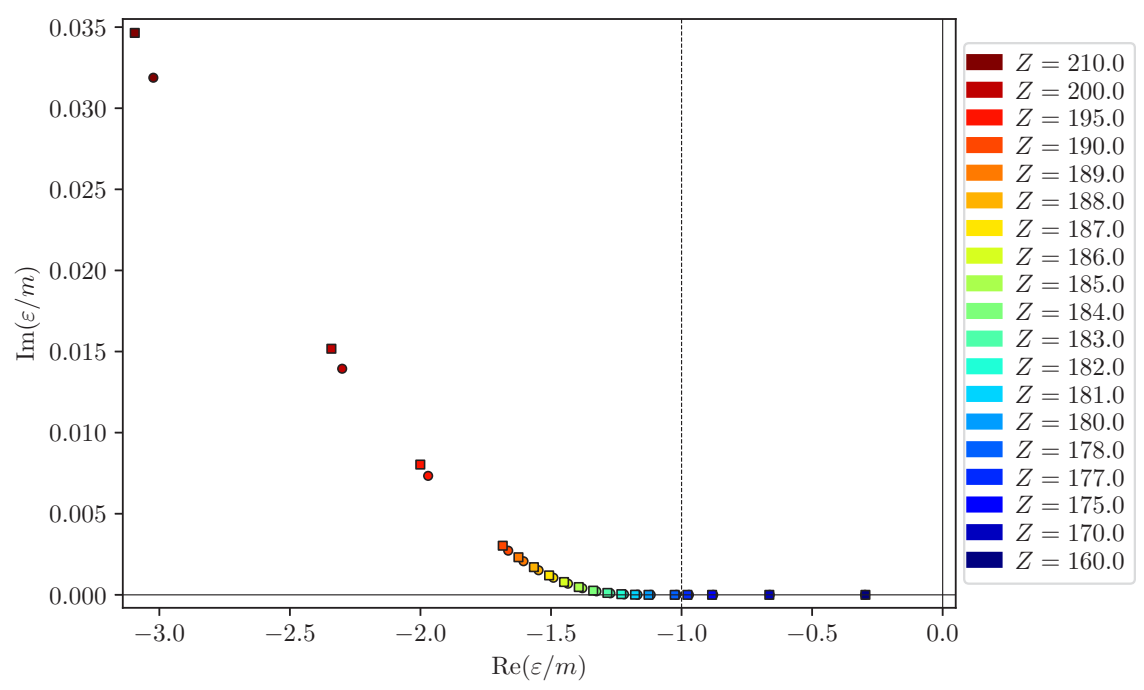

Figure 4: The dependence of the ground state energy on $Z$. The square markers are for the exact values of the energy and the round markers are for the approximate ones (see details in [4]). The correspondence between color and $Z$ is shown in the legend (the real part of the energy is monotonically decreasing). At $Z=Z_{\text {cr }}$ the bound states become resonances with positive $\operatorname{Im}[\varepsilon]$.

respect to positron emission to infinity, so it is an average time of $e^{+} e^{-}$pair production (in reality two independent pairs are produced because of electron spin degeneracy).

The potential barrier which holds the positron in the vicinity of the nucleus is shown in Fig. 2 of [1]; its penetration time is given by the analytical formulas $(4.14,4.15)$, and the results of numerical calculations are shown in Fig. 13 of the same review paper. We reproduced that curve for the dependence $\gamma(Z)$ for the energy of the Gamov (quasistationary) state, see Figure 4.

Let us finally mention that we agree with the description of the stable states of a supercritical nucleus made in Section 6 of [23]: empty states in the upper continuum, empty discrete levels, and occupied states in the lower continuum. The levels of the lower continuum that get occupied by electrons after the diving process form the so-called "charged vacuum"; it has charge $-n$, where $n$ is the number of these levels. The $n$ positrons that get emitted compensate for this negative charge. A supercritical nucleus is no longer naked and its electric charge is partially screened by these electrons.

Indirect evidence of such a phenomenon is found in graphene physics [24, 25].

\section{Conclusions}

The potential of the pointlike charge in a superstrong external magnetic field has been considered. The enhancement of radiative corrections leads to the screening of the potential. The results for these corrections were known from previous studies, but not in the all space. The modified potential was calculated numerically in all directions.

The influence of screening and the finite nucleus size on the energy levels of the hydrogen-like ions has been studied. Both screening and the finite nucleus size push the ground energy level up. The screening starts at $B \sim m^{2} / e^{3} \approx 6 \cdot 10^{15} \mathrm{G}$ and leads to the freezing of the ground energy level. The 
finite nucleus radius $R$ comes into play at $B \sim 1 /\left(e R^{2}\right)=10^{17} \div 10^{18} \mathrm{G}$ and the ground energy level goes up until in the magnetic fields $B \sim 1 /\left(e^{3} R^{2}\right)=10^{19} \div 10^{20} \mathrm{G}$ it reaches the final freezing energy. The main result is the calculation of the critical nucleus charge in the magnetic field shown in Fig. 3.

The physics of supercritical nuclei was re-examined focusing on the scattering phase and its dependence on the energy of the diving electronic level, for which both exact and approximate formulae were given. The arguments in favor spontaneous $e^{+} e^{-}$pairs production from a supercritical nucleus were provided.

\section{Acknowledgements}

S. Glazyrin is supported by RFBR under grants 16-32-00241. S. Godunov is supported by RFBR under grant 16-32-60115. M. Vysotsky is supported by RFBR under grant 16-02-00342.

\section{References}

[1] Ya.B. Zeldovich, V.S. Popov, UFN 105, 403 (1971)

[2] B. Müller, J. Rafelski, W. Greiner, Z. Phys. 257, 62 (1972)

[3] B. Müller, J. Rafelski, W. Greiner, Z. Phys. 257, 183 (1972)

[4] S.I. Godunov, B. Machet, M.I. Vysotsky, Eur. Phys. J. C 77, 782 (2017)

[5] V.N. Oraevskii, A.I. Rez, V.B. Semikoz, Sov. Phys. JETP 45, 428 (1977)

[6] P. Schlüter, G. Soff, K.-H. Wietschorke, W. Greiner, J. Phys. B: At. Mol. Phys. 18, 1685 (1985)

[7] A.E. Shabad, V.V. Usov, Phys. Rev. Lett. 98, 180403 (2007)

[8] A.E. Shabad, V.V. Usov, Phys. Rev. D 77, 025001 (2008)

[9] N. Sadooghi and A. Sodeiri Jalili, Phys. Rev. D 76, 065013 (2007)

[10] M.I. Vysotsky, Pis'ma Zh. Eksp. Teor. Fiz. 92, 22 (2010) [JETP Lett. 92, 15 (2010)]

[11] B. Machet, M.I. Vysotsky, Phys. Rev. D 83, 025022 (2011)

[12] S.I. Godunov, B. Machet, M.I. Vysotsky, Phys. Rev. D 85, 044058 (2012)

[13] S.I. Godunov, S.I. Glazyrin, JETP Lett. 105, 147 (2017)

[14] S.I. Godunov, M.I. Vysotsky, Phys.Rev.D 87, 124035 (2013)

[15] S.I. Godunov, Phys. Atom. Nucl. 76, 901 (2013)

[16] S.I. Glazyrin, S.I. Godunov, JETP Lett. 105, 147 (2017)

[17] V.V. Skobelev, Izv. Vyssh. Uchebn. Zaved., Fiz. 10, 142 (1975) [Sov. Phys. J. 18, 1481 (1975)]

[18] Yu.M. Loskutov, V.V. Skobelev, Phys. Lett. A 56, 151 (1976)

[19] A.E. Shabad, Sov. Phys., Lebedev Inst. Reps. 3, 11 (1976)

[20] D.B. Melrose and R.J. Stoneham, Nuovo Cimento A 32, 435 (1977)

[21] D.M. Gitman and A.E. Shabad, Phys. Rev. D 86, 125028 (2012)

[22] T.C. Adorno, D.M. Gitman, A.E. Shabad, Phys. Rev. D 93, 125031 (2016)

[23] V.M. Kuleshov, V.D. Mur, N.B. Narozhny, A.M. Fedotov, Y.E. Lozovik, V.S. Popov, Phys. Usp. 58, 785 (2015). [Usp. Fiz. Nauk 185, 845 (2015)]

[24] Y. Wang, D. Wong, A.V. Shytov, V.W. Brar, S. Choi, Q. Wu, H.-Z. Tsai, W. Regan, A. Zettl, R.K. Kawakami, S.G. Louie, L.S. Levitov, M.F. Crommie, Science 340, 734 (2013)

[25] J. Mao, Y. Jiang, D. Moldovan, G. Li, K. Watanabe, T. Taniguchi, M.R. Masir, F.M. Peeters, E.Y. Andrei, Nature Physics 12, 545 (2016) 